\title{
Index of names
}

Abbink, Mrs. 279

Abendanon, J.H. 192, 194, 222, 247 -

9, 259-60, 264, 272, 278, 299, 301

Abeyasekere, Susan 7

Alken, J. 82, 101, 103, 108

Amirati, Raden Adjeng 297

Amsterdamska, Olga 2

Arntzenius, A.K. 138

Asch van Wijk, jonkheer T.A.J. van 187

Asharie 199

Asmaoen, Mas 212

Astijem, Nji 138, 152, 160, 233, 311

Augustin, P.L. 175-6

Bakker, A.J.G.J.P. 296

Baud, J.C. 60, 62, 65, 68, 73, 77, 79, 117

Becking, B.E.J.H. 158, 225

Bervoets, H. 157, 232-3, 235-7, 242-

3, 244, 248, 258, 260, 265, 285

Bervoets-van Ewijck, L.L. 260-1, 285

Beukers, Harm xv

Beyers van de Vlugt, S. 263

Beynon, Hans 204

Bleeker, P. 19, 21, 28-9, 84, 91, 93, 95-6, 113, 117

Blokland, Nonja Van 279, 281

Boerma, N.A.J.F. 122

Boissevain, Charles 239, 241

Bommel, Teuntje van 139

Bontius 19-20
Boomgaard, Peter 7, 72

Boorsma, W.G. 21, 26, 276.

Borg, A.H. van der 122-3

Borgers, A.H. 7

Bosch, J. van den 54, 116

Bosch, W. 7, 33, 37, 60, 62-70, 73, 75-6, 78-81, 83-6, 88, 91, 93, 114, 118, 119-21, 126-8, 130, 133, 136, $143,145,147,251,269,319$

Bosscher, C. 158, 225

Brand, J. van den 290

Broekmeyer, J.G.X. 28, 160, 281

Brooshooft, P. 272

Bruyn, A. de 116

Buning, J.F. 44

Burg, C.L. van der 5, 17, 20, 24-5, 28, 35, 141, 276, 281

Buuren, H.B. van 232-6, 240, 243-8, 251, 253-6, 258-9, 261-3, 265-6, $274,277,311,314$

Bijker, J. 256

Casembroot, C.J. de 71

Cochius, F.D. 76, 84

Coen, Jan Pieterszoon 19

Cook, Harold 2

Coolsma, S. 284

Cornelissen, F.J. 140, 228

Cornet, F. 32

Cornets de Groot van Kraaijenburg, jonkheer J.P. 71 
Coronel, J. 287

Crawfurd, John 23

Credé, C.S.F. 235

Cremer, J.T. 167, 240, 243-4

Dengoean 32

Deventer, C.Th. van 201-2, 222, 272

Diponegoro, Prince 53

Dissel, J.A. van 21

Djajadiningrat, Pangeran Aria

Achmad 210

Djarisah 259-60

Djasminah 235-6, 245-6, 266

Djelantik, Madé 297

Djojohadikusumo, Raden Mas

Margono 207-8

Doeff, H. 282

Doel, H.W. van den 53, 59

Donders, F.C. 100

Doorn, J.A.A. van 4

Doyer, D. 44

Dubois, Eugène 31

Duymaer van Twist, A.J. 55, 79, 8022, 116

Eilerts de Haan, L.J. 180, 212, 293

Elson, R.E. 59

Engelenberg, A.J.N. 181-2

Engelmaijer, P. 192

Epp, F. 23-4

Eijkman, G. 6, 184, 188, 201, 293

Fisher, Alison 8

Fles, J.A. 100

Fraenkel, S.A. 72

Francis, E. 14

Fransen van de Putte, I.D. 55, 72, 95, $97,157,225$
Freytag, G.J. de 247

Fritze, E.A. 120

Gent-Detelle, Nonja Van 279, 281

Gersen, A.A. 194, 248, 264

Geyl, A. 122-3

Godefroy, F.J. 120-1, 127

Goelam 12, 198

Goltstein van Oldenaller, W. Baron van 158

Gonggrijp, G.L. (pseudonym

Opheffer) 297

Gorkom, W.J. van 194, 278

Gouda, Frances xv

Greiner, C.G.C.F. 11, 22, 25

Groenevelt, W.P. 229

Groneman, I. 44, 177

Grijns, G. 293

Hadjiwibowo 304

Haga, J. 11, 193, 264, 301

Harloff, G.H.G. 24, 42, 44, 121, 262

Haspels, A. 25

Hasselman, J.J. 99, 146, 153, 157

Heteren, Godelieve van 5

Heutsz, J.B. van 82

Hien, H.A. van 10, 16

Hoëvell, W.R. Baron van 70, 269

Hofhout, Johannes 39

Holle, Karel Frederik 210

Huizing, Anja 2

Idenburg, A.W.F. 203, 209, 218, 273, 284, 290

Ido, Victor see Wall, Hans van de Irwin, Graham 5

Iskandar, Purwanta 11

Ismael 177-8, 248, 285 
Jacob, F. s' 114

Jacobs, Aletta 218

Jenner, Edward 47

Jenner, Mark S.R. 3-4

Jordaan, Roy E. 6, 20

Junghuhn, Franz Wilhelm 31

Kartawinata 172

Kartini, Raden Adjeng 198, 247, 260

Kartodirdjo, Sartono 54

Kemp, P.H. van der 187-8, 230, 298

Kern, J.W.G. 248

Keuchenius, L.W.C. 230

Kielstra, E.B. 227-8

Kiewiet de Jonge, G.W. 181

Kleiweg de Zwaan, J.P. 10, 42, 275

Klinkert, H.C. 16

Kloppenburg-Versteeg, J. 279-81

Koerong, Alida 139, 142

Kohlbrugge, J.H.F. 11, 48, 191, 194, 248, 258, 266, 274-5, 279, 281, 294-6, 298-300, 303, 305-6

Kol, H. van 184, 187, 189, 240, 258, 261, 290, 311

Koppeschaar, H. 278

Kreemer, J. 16, 19, 23, 24, 262

Kreemer Jr., J. 11, 17, 19, 24

Kuenen, W.A. 289

Kuyper, Jo 286

Kwast, J. 274-5, 306

Kworo, Raden Mas Noto 198, 263

Legge, J.D. 53

Liem 297

Lim, N.F. 212

Lindeboom, G.A. 1

Lindgreen, J.J. 23, 44

Lokhorst, H. van 188, 228-30
Loudon, J. 113, 157

Luchtmans, G. 48, 106, 225

Ludeking, E.W.A. 137

Lulofs, Saar 282

Maasland, H.F.P. 237, 241

McKeown, Thomas 1

Mandt, C.C.W. 39

Mangkoepradja, Raden Moehamad

Saleh 197, 264

Mangoenkoesoemo, Tjipto 199, 204

Markati 236-7, 266, 311

Marland, Hilary 7

Mausbach, Wilhelmina 139

Mayer, L.Th. 16, 275

Meijer, E.F. 29

Moekadi, Raden 200

Monnikendam, S. 239, 241

Moore, G. van der 108, 142

Moro, Si 199

Moulin, D. de 7

Münnich, Juriaan 31

Muller, G.H. 92, 142

Munnick, L.W.H. de 63, 72

Munnick, O.M. de 57

Mijer, P. 101, 109-10

Neisser, A. 181

Nieuwenhuys, Rob 279

Noodt, U.J.G. 228

Noor, Ma 262

Opheffer see Gonggrijp, G.L.

Pakoe Alam VI, Pangeran Adipati

Ario 297

Pauw, W. 174, 239, 243, 264

Permadi 199 
Peverelli, P. 122, 289

Ploem 39

Prang Wadhono, Pangeran Adipati 50

Prawiro Atmodjo, Mas 113

Pruijs, H.S. 286

Pruys van der Hoeven, A. 32, 78, 95-7, 107, 182

Radjiman 203

Raie, Mas 48

Redeker-Pulle, A.J. 131, 147

Reiche, M.Th. 112, 156, 158, 278

Reinwardt, C.G.C. 119

Reverby, Susan 1

Rhemrev, J.L.T. 290

Ricklefs, M.C. 53, 59

Riemsdijk, J.J.W.E. van 112

Rienks, Adriaan S. 11

Rivai, Abdoel 201-2, 256

Robberts, Johanna 139

Rochussen, J.J. 60, 62, 65-73, 75-6, 79, 115

Rodenwalt, E.R.K. 182

Roll, H.F. 168, 185, 188, 193-4, 215-6

Roorda van Eysinga, S.E.W. 71

Rosner, David 1

Ruijter, H.C.K.Th. de 303

Samallo, Jacob 193-4

Sambang 10, 17

Samgar 115, 201

Sampan 153

Samir 199

Sankum 233

Sariëm 138, 140, 152-3, 228

Schaddelee-Hoogvelt, J. 46
Scheer, A. van der 179-80

Scheurer, J.G. 284, 286

Schoute, D. 6

Schüffner, W.A.P. 289

Schülein, J. 275, 294

Schuler, Ms 21

Schultz, A. 29

Sciortino, Rosalia 7

Sigerist, Henry 1

Sima 139, 142

Sitanala 184

Sloet van de Beele, L.A.J.W. Baron 95

Smyth, Ines 7

Snouck Hurgronje, C. 41, 171, 1767, 186, 200, 209-10, 212

Soedirohoesodo, Mas Wahidin 204, 210

Soekarno 201

Soemeroe, Raden 196-7

Soeradji, Mas 208

Soeriadarma 199

Soerjatin, Raden 189

Soerjo, Raden Mas Tirto Adhie 170, 208

Soetomo, Raden 6, 172, 177, 208,

215

Sprenger van Eyk, J.P. 227-8

Stein, Eric A. 126

Steiner, L. 291

Stibbe, F.S. 253, 256

Stok, N.P. van der 12

Stokvis-Cohen Stuart, N. 211, 257, 261, 297, 302

Stoll, Mrs. 279

Stortenbeker, W. Jr. 113

Stratz, C.H. 23-5, 31, 237-9, 242, 245, 254, 276 
Suparlan, Parsudi 6

Swieten, J. van 95-7, 182

Székely-Lulofs, Madelon 275, 290

Taroenadibrata, Raden Goeteng 174, 191, 195, 209, 210

Taslim 111

Tasminten 245-6, 266

Tehupeiory, J.E. 177, 196, 203, 253

Tehupeiory, W.K. 172, 175-6, 185, 196, 203, 211-3, 215, 222, 253

Thomas, Marie 220

Thorbecke, J.R. 54, 93, 100, 122

Tjo Kim Bin 277

Toer, Pramoedya Ananta 208

Tombrink, E.P. 100, 110

Tonggo, Ma 21

Treub, H. 238

Verdoorn, J.A. 7

Verwey, L.H. 93

Veth, Bas 298, 304

Veth, P.J. 21

Visscher, C. 60, 62

Vogel, W.Th. de 172, 208, 250, 264, 275, 291

Vogelpoel, Th.G. van 193

Vorderman, A.G. 5, 18, 19-20, 45, 146, 152, 179, 247, 262, 277, 281, 293

Vortisch van Vloten, H. 45

Vries-Bultman, C. de 264
Waal, E. de 101

Waal, L.W.J. de 99, 102, 109-10, 112, 146, 153-8

Waart, A. de 191, 220-1

Waitz, F.A.C. 20, 22, 31

Wall, Hans van de (pseudonym Victor Ido) 82

Wallis, Patrick 3-4

Walraven, Willem 53

Warouw, Anna 220

Wassink, G. 20, 30, 83, 89-90, 92-7, 101, 121, 130, 133-4, 136-7, 139, 144-5, 148, 281

Waszklewicz, A.E. 47, 97, 99, 102, $111-2,135,150,153-6$

Weitzel, A.W.P. 138

Westhoff, C.H.A. 300

Westplat, J. 201, 213

Wilhelmina, Queen 272

Wilken, G.A. 275

William I, King 54

William III, King 99

Winkler, C. 191, 215, 248, 256

Woodward, Mark 6

Wijchgel, G.J. 235

Wijck, Jonkheer C.H. van der 244

Wijck, Jonkheer O. van der 243-4

Zainal 198, 287

Zembsch-de Klemp, H.J. 131, 150, 225 